\title{
Comparative Study of CouchDB and MongoDB - NoSQL Document Oriented Databases
}

\author{
Niteshwar Datt Bhardwaj \\ Centre for Development of \\ Advanced Computing (C-DAC), \\ Mohali \\ Punjab Technical University, \\ Jalandhar - Kapurthala Highway, \\ Kapurthala 144601 (Punjab), India
}

\begin{abstract}
A comparison between MongoDB and Apache CouchDB keeping the data and other environments same using Java programming language and Apache JMeter confirms that the MongoDB document write rate is many times faster than the Apache CouchDB.
\end{abstract}

\section{General Terms}

Performance, Reliability

\section{Keywords}

SQ1, NoSQL, Database, MongoDB, CouchDB, Big Data.

\section{INTRODUCTION}

In this paper, it is purported to compare the behaviour of NoSQL document oriented databases. There are number of document based NoSQL databases available, but all have different mechanisms to store the data in document format.

The comparisons are important as they provide overview of usage of NoSQL databases as per the user requirements.

The methodology envisages the use of Java programming language to highlight the comparative results for two widely used document oriented databases - MongoDB and Apache CouchDB.

\section{NoSQL DATABASES}

There are various document oriented NoSQL databases available, both open source and licensed. However it is a rigorous proposition to decide which is to be used and when. So there is need for performance comparison of various document oriented NoSQL databases.

The leading document oriented NoSQL databases are: MongoDB , CouchDB, Couchbase, Terrastore, RavenDB. OrientDB. This paper however covers the comparison of MongoDB and CouchDB.

\section{COMPARITIVE ANALYSIS AND RESULTS}

For comparing the insertion rate (processing time), read / write operations of two leading NoSQL document-oriented databases - the MongoDB and CouchDB, the object oriented programming language, Java, with performance measuring tool Apache JMeter, is used. Some performance evaluation tests have been carried out. Though the database sizes used for the analysis are comparatively smaller, a clear difference in various factors of comparison has been observed. The environment used for conducting these tests was same for both MongoDB and CouchDB.
Table 1. Benchmarking parameters

\begin{tabular}{|l|l|ll|}
\hline Sr. No. & Entity & Value \\
\hline 1. & $\begin{array}{l}\text { Operating } \\
\text { System }\end{array}$ & Windows 7 (64 bit architecture) \\
\hline 2. & RAM & $8 \mathrm{~GB}$ \\
\hline 3. & $\begin{array}{l}\text { Document } \\
\text { used }\end{array}$ & $\begin{array}{l}\text { JSON with approximate 160 } \\
\text { bytes }\end{array}$ \\
\hline
\end{tabular}

Table 2. Databases and Tools Configuration

\begin{tabular}{|l|l|l|}
\hline Sr. No. & Entity & Value \\
\hline 1. & MongoDB & 2.6 .3 \\
\hline 2. & Apache CouchDb & 1.6 .1 \\
\hline 3. & Apache JMeter & 2.13 \\
\hline 4. & $\begin{array}{l}\text { Mongo VUE - } \\
\text { GUI Tool for } \\
\text { MongoDB }\end{array}$ & 1.6 .9 .0 \\
\hline 5. & $\begin{array}{l}\text { Java } \\
\text { Futon on Apache } \\
\text { CouchDB 1.6.1 }\end{array}$ & $\begin{array}{l}\text { Springsource Tool } \\
\text { Suite (Eclipse for } \\
\text { Java) }\end{array}$ \\
\hline 7. & 3.6 .4 \\
\hline
\end{tabular}

The methodology followed for comparing the databases is:

1. Define the configurations

2. Install the databases

3. Define the data set to be used

4. Write Java programs to connect with both the databases and insert the defined volume of data (document) in both databases individually.

5. Measure the insertion rate / response time using Apache JMeter

6. Analyze the results by plotting graphs and charts.

The loads used for the activity are:

1) 100 users and no loop, 100 samples, one by one execution for CouchDB and MongoDB; and

2) 10 users and 10 as loop, 100 samples, one by one execution for CouchDB and MongoDB. 
The charts and results for the tests conducted are depicted below:

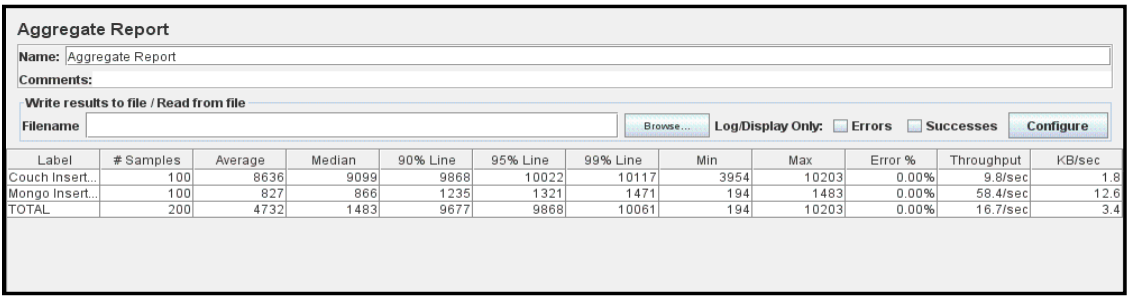

Fig 1: The aggregate report for insertion rate for CouchDB and MongoDB.

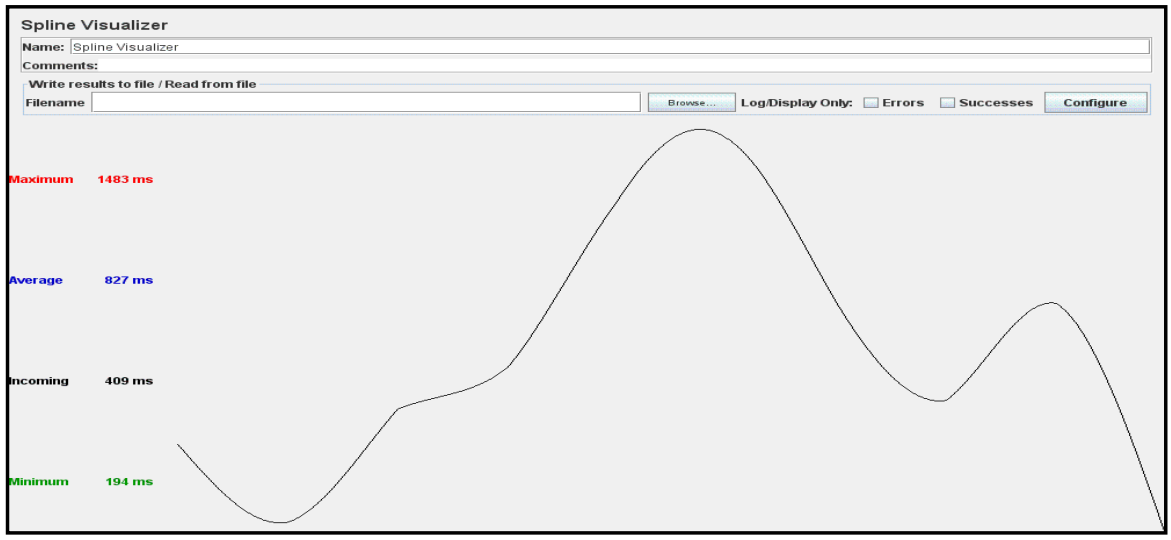

Fig 2: The Spline Visualizer for MongoDB document insertion test.

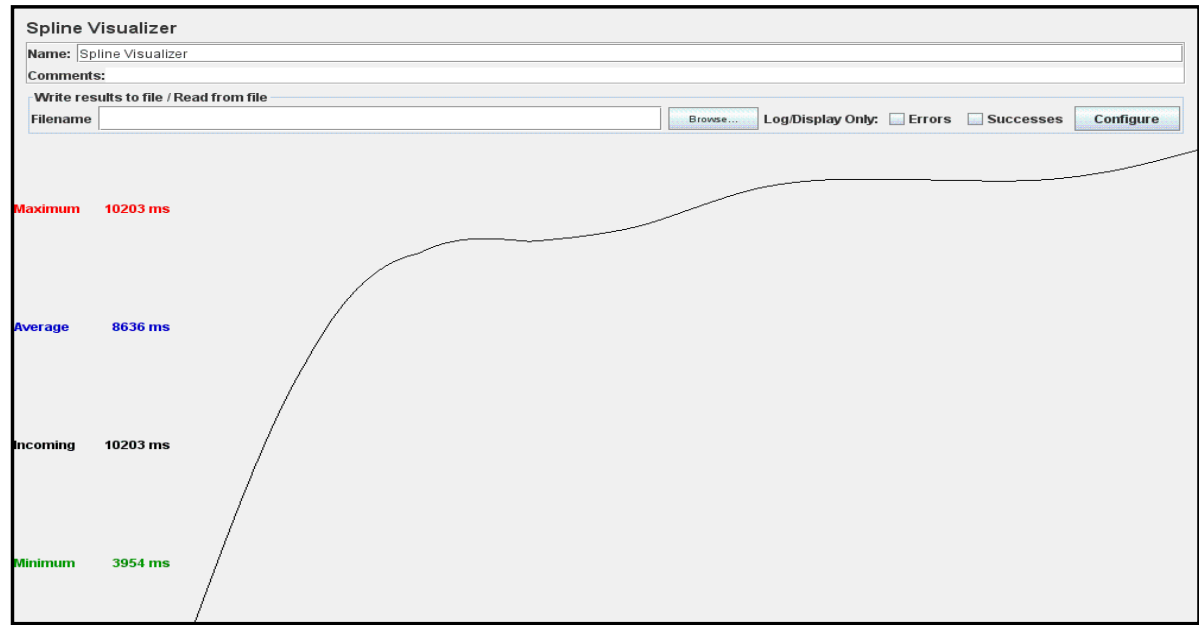

Fig 3: The Spline Visualizer for CouchDB document insertion test.

\section{CONCLUSION}

The average response time for MongoDB is $827 \mathrm{~ms}$ with throughput of 58.4per sec. for 100 samples.

The average response time for CouchDB is $8636 \mathrm{~ms}$ with throughput of 9.8 per sec. for 100 samples.

In MongoDB, the document insertion rate is approximately 10 times better than Apache Couch DB under the stated conditions and environment.

The performance can be more generalized defining the nodes for the server and replication for MongoDB and CouchDB.

\begin{tabular}{|c|c|c|}
\hline Type & $\begin{array}{c}\text { Collection } \\
\text { Oriented }\end{array}$ & $\begin{array}{c}\text { Schema Free - Flat } \\
\text { Address Space }\end{array}$ \\
\hline $\begin{array}{c}\text { DSON - } \\
\text { "Binary }\end{array}$ & $\begin{array}{c}\text { Serialized } \\
\text { dOcument } \\
\text { Notation" } \\
\text { format }\end{array}$ & $\begin{array}{c}\text { JSON - "JavaScript } \\
\text { Object Notation" } \\
\text { format. }\end{array}$ \\
\hline Protocol & Custom & HTTP \\
\hline $\begin{array}{c}\text { Response } \\
\text { Time }\end{array}$ & Faster & $\begin{array}{c}\text { Slower as compared } \\
\text { to MongoDB }\end{array}$ \\
\hline
\end{tabular}

Table 3: Comparison MongoDB vs. CouchDB

\begin{tabular}{|c|c|c|}
\hline Parameter & MongoDB & Apache CouchDB \\
\hline Installation & $\begin{array}{c}\text { Easy with } \\
\text { shell utilities }\end{array}$ & $\begin{array}{c}\text { Easy and Fast with } \\
\text { web utilities }\end{array}$ \\
\hline
\end{tabular}




\section{ACKNOWLEDGMENTS}

Creation of a paper like this one requires a great deal of effort, hard work and blessings of many great people and to name everyone is beyond simply beyond reach who directly or indirectly guided in the preparation of this paper. However two organizations with whom I am connected directly need special mention.

First and foremost it is a matter of great pleasure to express my gratitude to Centre for Development of Advanced Computing, Mohali for granting me this lifetime experience which I shall always treasure. Secondly, I am extremely indebted to Infosys Ltd. for giving me an opportunity to explore a new dimension of the upcoming technologies and providing me this brilliant opportunity.

\section{REFERENCES}

[1] Zhang, Shaolong ; Network Center, Zhejiang Radio \& Television University, Hangzhou, 310012, China, "Application of document-oriented NoSQL database technology in web-based software project documents management system," in IEEE International Conference on Information Science and Technology (ICIST), Yangzhou, March 2013.

[2] Gansen Zhao, Weichai Huang, Shunlin Liang, Yong Tang, "Modeling MongoDB with Relational Model," IEEE Fourth International Conference on Emerging Intelligent Data and Web Technologies (EIDWT), Xi'an, September 2013.
[3] Dharmasiri, H.M.L. and Goonetillake, M.D.J.S., "A federated approach on heterogeneous NoSQL data stores," in IEEE International Conference on Advances in ICT for Emerging Regions (ICTer), Colombo, December 2013.

[4] Phan Thi Anh Mai, Nurminen, J.K., and Di Francesco, M., "Cloud Databases for Internet-of- Things Data" IEEE International Conference on Internet of Things(iThings), 2014 and Green Computing and Communications (GreenCom), September, 2014.

[5] The Altoros, "The NoSQL Technical Comparison," publication on Couchbase

[6] Julien Carme, R\&D engineer, Atos Worldline and Francisco José Ruiz Jimenez, Technical Architect Manager, Atos Spain, "Open Source Solutions for Big Data Management," Atos.

[7] The United Software Associates, "Comparative benchmarks: mongodb vs. Couchbase vs. Cassandra", MongoDB, March 2015.

[8] The United Software Associates, "Scalability Benchmarking: MongoDB and NoSQL Systems" MongoDB, June 2015. 